\title{
Optimization of optical parameters for quantum information processing inside photonic crystals
}

\author{
Hiroyuki Nihei ${ }^{1}$ and Atsushi Okamoto ${ }^{2}$ \\ ${ }^{1}$ Health Sciences University of Hokkaido, Hokkaido 061-0293, Japan; nihei@hoku-iryo-u.ac.jp \\ ${ }^{2}$ Hokkaido University, Kita-ku, Sapporo, Hokkaido 060-0814, Japan; ao@optnet.ist.hokudai.ac.jp
}

\begin{abstract}
We have optimized the control laser strength to carry out quantum information processing inside photonic crystals, by calculating the time-averaged probabilities of finding an atom on excited states encoding the quantum information.
\end{abstract}

\section{INTRODUCTION}

Quantum computation using a quantum state of an atom embedded in photonics is one of the challenging goals in the area of the physics and theory of nanostructures. A significant issue in this field is to clarify the suppression of quantum information loss in quantum computing. Near the embedded atom, light is confined due to the presence of a photonic band gap (PBG) [1, 2]. This suppresses atomic decay, leading to the formation of a nonzero steady-state atomic population on excited states, which is a key mechanism of suppressing quantum information loss. In addition, the processing of quantum information is necessary for quantum computing. This requires a periodic oscillation of populations in excited states encoding quantum information [3]. Even in the oscillation, the suppression of quantum information loss is also necessary for quantum computing; however, for the oscillation of populations, the localized mode should be moved toward the edge frequency of the PBG [4], so that the loss is unavoidable in quantum information processing.

In this paper, we study the optimum optical design for suppressing quantum information loss in processing quantum information encoded by excited states of an atom embedded in photonic crystals by calculating the time-averaged probabilities of finding the atom on excited states, where this calculation enables comprehensive analysis of several types of quantum state including the steady-state population and the oscillation of populations.

\section{QUANTUM INFORMATION PROCESSING}

The atom is assumed to have a three-level energy configuration, where a coherent superposition of the two upper levels,

$$
|\psi(t)\rangle=a_{0}(t)|0\rangle+a_{1}(t)|1\rangle
$$

is used for encoding quantum information and acts as a qubit. One of the transition frequencies of the atom is assumed to be far inside the PBG, which guarantees the light confinement effect. The other transition frequency is assumed to be near the edge of the PBG, where a control laser is used for coupling the two upper levels that encode quantum information. The ratio of the superposition $\tan \theta(t)=\left|a_{0}(t)\right| /\left|a_{1}(t)\right|$ can be changed by applying an ultrashort pumping laser pulse onto the atom. We consider the time when the atom is pumped up as the initial time of this system, i.e, $t=0$. Depending on the initial ratio $\theta(0)$ determined in $|\psi(0)\rangle$, the quantum state of the atom in the long-time limit $(t \rightarrow \infty)$ shows a steady-state population on the two upper levels, a periodic oscillation of populations, or their intermediate states [4]. Their combination is used for storing and processing of quantum information encoded by the two upper levels $|0\rangle$ and $|1\rangle$.

\section{TIME-AVERAGED PROBABILITIES}

The time-averaged probability of finding the atom on excited states encoding quantum information is given by

$$
(1 / T) \int_{0}^{T} \lim _{t \rightarrow \infty}\left\{\left|a_{0}(t)\right|^{2}+\left|a_{1}(t)\right|^{2}\right\} d t
$$

which can be applied to several types of quantum states. Figure 1 shows the time-averaged probability using a polar coordinate, where the angular coordinate shows various initial ratios $\theta(0)$. Here, the more the probability is enhanced, the more the loss is suppressed.

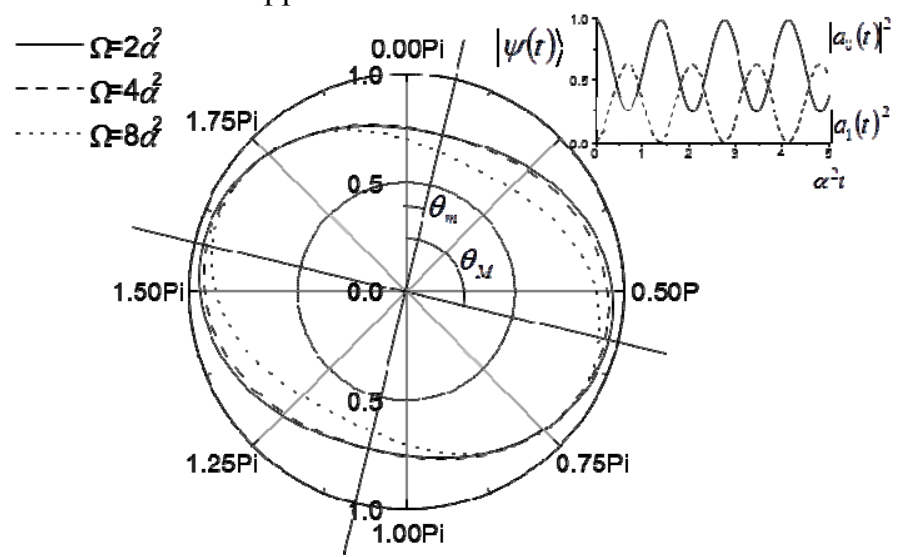

Fig. 1: Time-averaged probabilities of the excited states of the atom using a polar coordinate, where $\alpha^{2}$ is a scaled parameter [2].

As shown in Fig. 1, the time-averaged probabilities are given by an ellipse. For the solid curve $\left(\Omega=2 \alpha^{2}\right)$, the ratios $\theta_{M}$ and $\theta_{m}$ give the major and minor axes, respectively. The time-averaged probability reaches a maximum at $\theta_{M}$, where the loss is strongly suppressed. When the initial ratio $\theta(0)$ is $\theta_{M}$, the atomic states $|\psi(t)\rangle$ show the steady-state population. On the other hand, the time-averaged probability is minimum at $\theta_{m}$, where the loss is enhanced. When the 
initial ratio $\theta(0)$ is $\theta_{m}$, the atomic states show a periodic oscillation of populations, as shown in the inset of Fig. 1. Here, the localized mode is moved to be near the edge frequency of the PBG for the formation of a population transfer path between excited states, so that the loss is unavoidable in the case of the oscillation of populations.

Furthermore, we find that this unavoidable loss depends on the control laser strength $\Omega$, where the loss is increased (namely, the time-averaged probability is decreased) by increasing the control laser strength $\Omega$, as shown by the dashed $\left(4 \alpha^{2}\right)$ and dotted $\left(8 \alpha^{2}\right)$ curves in Fig. 1. This is because a strong control laser leads to a wide Rabi splitting so that the transition frequency approaches the edge frequency of the $\mathrm{PBG}$, leading to the atomic decay to the ground state. Therefore, the loss is suppressed by decreasing control laser strength, but such a weak control laser may cause only a weak oscillation of populations; a strong oscillation is required for processing quantum information.

\section{OPTIMIZATION OF OPTICAL PARAMETERS}

We reveal the optimum control laser strength for carrying out a sufficiently large suppression of quantum information loss and a sufficiently large oscillation of populations to achieve quantum information processing. In Fig. 2, we show the time-averaged probabilities at the major and minor axes as a function of control laser strength. Here, despite a slight decrease in the time-averaged probability at the minor axis (a dashed curve) near $\Omega=0$, the quantum information loss is strongly suppressed for a weak control laser of $\Omega<5 \alpha^{2}$. However, for a strong control laser of $\Omega>9 \alpha^{2}$, although the time-averaged probability at the major axis $\left(\theta(0)=\theta_{M}\right.$, the atom showing the steady-state populations) maintains a high value, the time-averaged probability at the minor axis ( $\theta(0)=\theta_{m}$, the atom showing the oscillated populations) sharply decays to zero, because of the wide Rabi splitting.

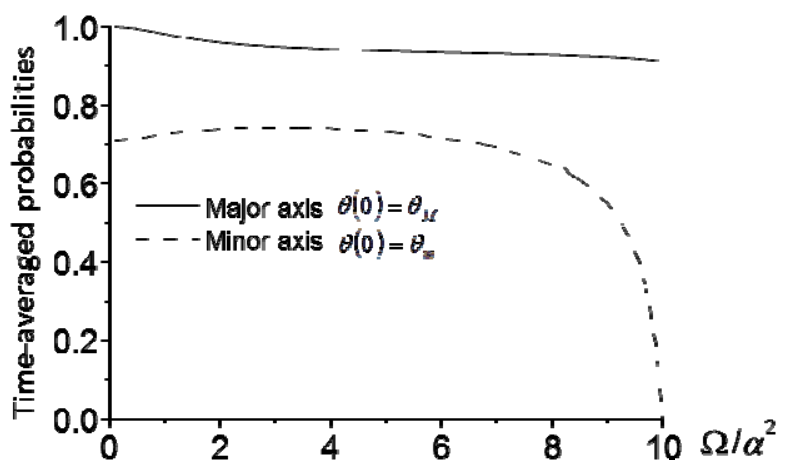

Fig. 2: Time-averaged probabilities at major (solid curve) and minor (dashed curve) axes as a function of control laser strength.

In Fig. 3, we show the rate of the populations oscillated between the two excited states $|0\rangle$ and $|1\rangle$ for the initial ratio $\theta(0)=\theta_{m}$. For example, the figure shows that, at $\Omega=5 \alpha^{2}, 80 \%$ of the populations are oscillated between the two excited states, $14 \%$ are oscillated in the ground state $|g\rangle$ and excited states, and $6 \%$ remain in their original state (not oscillated with other states). The rate of the populations oscillated between the two excited states is decreased by decreasing control laser strength. Particularly for $\Omega<3 \alpha^{2}$, this rate is sharply decreased. For example, for $\Omega=\alpha^{2}$, only $30 \%$ of the populations are oscillated between excited states. When $\Omega=0$ (no control laser), the population transfer path is vanishes, so that the oscillation is stopped.

As a result of Figs. 2 and 3, we find that, for $\Omega=4 \alpha^{2} \sim 5 \alpha^{2}$, both of a sufficiently large suppression of the quantum information loss and a sufficiently large oscillation of populations are carried out. In this case, $70 \%$ of the populations are kept even in the minor axis, as shown in Fig. 3, and $80 \%$ of the populations are oscillated in the excited states encoding quantum information.

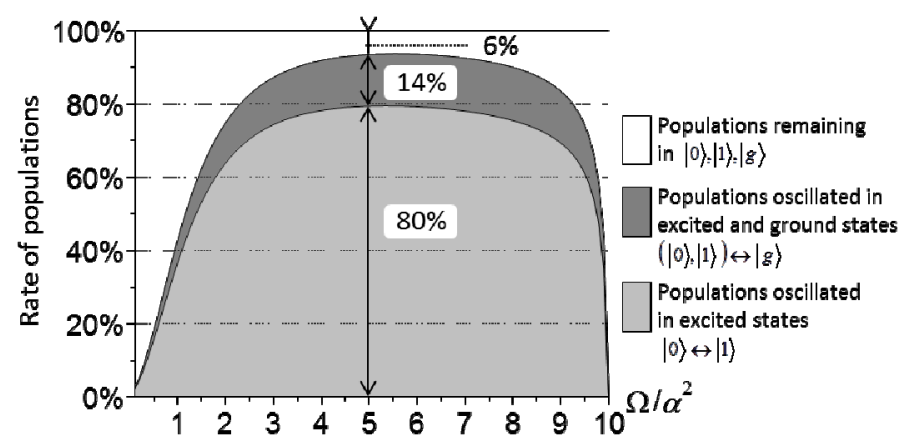

Fig. 3: Rate of populations oscillated in excited states as a function of control laser strength.

\section{CONCLUSION}

We have optimized the control laser strength to carry out quantum information processing inside photonic crystals. In this demonstration, we have calculated the time-averaged probabilities of excited states of an atom embedded in photonic crystals. The result revealed an optimum control laser strength for carrying out a sufficiently large suppression of quantum information loss and a sufficiently large oscillation of populations to achieve quantum information processing. Finally, we have found that the control laser strength $\Omega=4 \alpha^{2} \sim 5 \alpha^{2}$ provides an optimum case, where more than $70 \%$ of the populations are maintained and $80 \%$ are oscillated in excited states encoding quantum information. These results of this study are useful for determining the designs of future quantum logic gates based on solid-state photonic crystal systems.

\section{REFERENCES}

[1] S. John, "Light control at will," Nature, vol. 460, p. 337, 2009.

[2] M. Woldeyohannes and S. John, "Coherent control of spontaneous emission near a photonic band edge: A qubit for quantum computation," Phys. Rev. A, vol. 60, p. 5046, 1999.

[3] H. Nihei and A. Okamoto, "Coherent control of excited atomic states inside a three-dimensional photonic bandgap", J. Mod. Opt., vol. 55, pp. 2391-2399, 2008.

[4] H. Nihei and A. Okamoto, "Confined light composed of a single localized mode inside photonic crystals for a qubit", J. Opt. and Quant. Electron., 44, 3-5, pp. 265-271, 2012. 